\title{
Models of particle acceleration in galaxy clusters by MHD turbulence
}

\author{
G. Brunetti \\ INAF Istituto di Radioastronomia, via P. Gobetti 101, I-40129, Bologna, Italy \\ email: brunetti@ira.inaf.it
}

\begin{abstract}
Present radio data provide indirect evidence that diffuse radio emission in the central cluster regions may originate from turbulent-acceleration of relativistic particles. I was invited to discuss models of particle acceleration by MHD turbulence in clusters and in these pages I briefly touch the main points of my talk.
\end{abstract}

Keywords. acceleration of particles, radiation mechanisms: nonthermal, turbulence, galaxies: clusters: general

\section{Introduction}

Galaxy clusters have a key role in the hierarchy of the large scale structure as they are the largest bound objects in the Universe, where most of the gravitational energy is dissipated. The discovery of Mpc-scale diffuse synchrotron sources in a fraction of galaxy clusters demonstrates that the Inter-Galactic-Medium (IGM) is magnetised at $\mu \mathrm{G}$ level and that relativistic particles are mixed with the thermal IGM (e.g. Carilli \& Taylor 2002). Synchrotron emission provides unique diagnostic of the large scale magnetic fields and relativistic particles in these environments and is a probe of the complex physical processes in the IGM (e.g. Rudnick et al 2009). Observations support a connection between diffuse synchrotron emission and cluster mergers, suggesting that a fraction of the energy dissipated during mergers is channelled into non-thermal components in the IGM (e.g. Enßlin et al. 1998; Cassano \& Brunetti 2005; Pfrommer et al. 2006).

Giant radio halos are the most spectacular examples of non-thermal phenomena in the IGM (e.g. Ferrari et al 2008). These diffuse synchrotron sources are typically centered on the Xray emitting region of galaxy clusters and extend for Mpc-scales. They can originate from secondary electrons injected during proton-proton collisions (e.g. Blasi \& Colafrancesco 1999), or by relativistic electrons re-accelerated by MHD turbulence generated during cluster-cluster mergers (e.g. Brunetti et al 2001; Petrosian 2001).

\section{Stochastic particle acceleration and evolution of radio halos}

We believe that turbulence in galaxy clusters is sub-sonic but strongly super-Alfvénic and that the main source of turbulence-dissipation is the collisionless damping on thermal electrons and protons. Yet a fraction of the turbulent energy can be channelled into the re-acceleration of pre-existing relativistic particles (e.g. Brunetti \& Lazarian 2007). Stochastic particle acceleration by MHD turbulence is rather inefficient in the IGM, that implies a maximum energy for the re-accelerated electrons of a few $\mathrm{GeV}$, due the short electron-cooling time. This is expected to cause a steepening at higher frequencies in the spectrum of turbulence-driven radio halos that is a key-feature of the turbulent scanario and allows to provide indirect evidences in favour of this mechanism (e.g. Thierbach et al. 2003; Brunetti et al. 2008).

In this scenario the formation and evolution of giant radio halos is complex and tightly connected with the evolution of the hosting clusters (e.g. Cassano \& Brunetti 2005). Present data suggest that radio halos are generated only in a fraction of massive and merging clusters and that they are "suppressed" when clusters become more dynamically relaxed, in a time-scale $<1$ Gyr. This short time-scale is most likely due to the fast cooling of the emitting electrons as soon as cluster-turbulence is dissipated, although magnetic field dissipation in the emitting regions might play a role (Brunetti et al 2009). Calculations that consider the acceleration of 
relativistic particles (protons and secondary electrons due to proton-proton collisions in the IGM) by merger-driven turbulence, show that radio halos and hard X-ray emission are expected in massive galaxy clusters only in connection with merging events, while gamma ray emission, at some level, should be long-living and more common in clusters (e.g. Brunetti 2009).

\section{LOFAR : unveiling radio halos in galaxy clusters}

The steep spectrum of radio halos makes these sources ideal targets for observations at low radio frequencies (e.g. Enßlin \& Röttgering 2002). In addition if turbulence plays a key role in accelerating relativistic particles in radio halos, very steep spectrum halos should be more common in the universe and would show up preferentially at these lower frequencies (e.g. Cassano et al. 2006).

The Low Frequency Array (LOFAR) promises an impressive gain of two orders of magnitude in sensitivity and angular resolution over present instruments in the frequency range 15-240 $\mathrm{MHz}$, and as such will open a new observational window on the Universe. LOFAR is expected to provide a leap forward in our understanding of the origin and evolution of the relativistic matter and magnetic fields in galaxy clusters. The discovery of about 400 giant radio halos at redshift $\leqslant 0.7$ is expected from LOFAR surveys at $120 \mathrm{MHz}$, most of them with very steep spectra (Cassano et al. 2009).

\section{Acknowledgements}

Support through grants ASI-INAF I/088/06/0, PRIN-INAF2007 and PRIN-INAF2008 is acknowledged.

\section{References}

Blasi, P. \& Colafrancesco, S., 1999, APh 12, 169

Brunetti, G., 2009, RMxAC 36, 201

Brunetti, G., Setti, G., Feretti, L., \& Giovannini, G., 2001, MNRAS 320, 365

Brunetti, G. \& Lazarian, A., 2007, MNRAS 378, 245

Brunetti, G., et al. 2008, Nature 455, 944

Brunetti, G., Cassano, R., Dolag, K., \& Setti, G., 2009, A\&A in press; arXiv:0909.2343

Carilli, C. L. \& Taylor, G. B., 2002, ARA\&A 40, 319

Cassano, R. \& Brunetti, G., 2005, MNRAS 357, 1313

Cassano, R., Brunetti, G., \& Setti, G., 2006, MNRAS 369, 1577

Cassano, R., Brunetti, G., Röttgering, H., \& Brüggen, M., 2009, A\&A in press; arXiv:0910.2025

Enßlin, T. A., Biermann, P. L., Klein, U., \& Kohle, S., 1998, A\&A 332, 395

Enßlin, T. \& Röttgering, H., 2002, A\&A 396, 83

Ferrari, C., Govoni, F., Schindler, S., Bykov, A. M., \& Rephaeli, Y., 2008, SSRv 134, 93

Petrosian, V., 2001 ApJ 557, 560

Pfrommer, C., Sppringel, V., Enßlin, T. A., \& Jubelgas, M., 2006, MNRAS 367, 113

Rudnick, L., et al. 2009, arXiv:0903.0824

Thierbach, M., Klein, U., \& Wielebinski, R., 2003, A\&A 397, 53 